\title{
Dietary omega-3 polyunsaturated fatty acids and treatment of cancer
}

\begin{abstract}
The results of animal studies have shown that the consumption of omega- 3 fatty acids can reduce the inflammatory biomarkers, enhance the efficacy of chemotherapy and decrease the side effects of the chemotherapy or of the cancer. Studies in human populations have associated high intake of fish or fish oil to decreased risk of some cancers. In this review we discuss the reasons for using omega-3 polyunsaturated fatty acids as adjuvant to ameliorate the efficacy of anticancer treatment.
\end{abstract}

Keywords: omega-3, cancer treatment, diet
Volume 8 Issue 3 - 2018

\author{
Behnaz Abiri,' Mohammadreza Vafa ${ }^{2}$ \\ 'Department of Nutrition, Ahvaz Jundishapur University of \\ Medical Sciences, Iran \\ ${ }^{2}$ Department of Nutrition, Iran University of Medical Sciences, \\ Iran
}

Correspondence: Mohammadreza Vafa, Department of Nutrition, School of Public Health, Iran University of Medical Sciences, Tehran, Iran, Email rezavafa@yahoo.com

\section{Introduction}

Global cancer incidence and mortality rates are high and elevating. Worldwide, it is estimated that the annual number of cancer diagnoses will promote to 22 million by $2032 .{ }^{1}$ In spite of progress made in cancer chemotherapy, advanced solid tumors consisting of advanced carcinomas, sarcomas, melanoma, and glioblastoms, yet show wide problems in their treatment and traditional therapeutic agents alone have not produced satisfactory long-term clinical outcomes. ${ }^{2,3}$ Also, in those cases which the tumor has seemingly been eliminated fully, micro-metastases of inactive tumor cells often result to relapse and to therapeutic failing. Hence, considering the problems of scape and survival to cancer progress, most oncologists have concluded that no unique therapy is enough to treatment of cancer. ${ }^{4,5}$

Dietary fish oil has been demonstrated to have useful impacts on some chronic degenerative disease like cancer. ${ }^{6}$ The useful impacts of fish oil appear to be resulted from its high content of the omega-3 fatty acids such as eicosapentaenoic acid (EPA) and docosahexaenoic acid (DHA). ${ }^{6}$ Some investigations have shown the ability of EPA and DHA to affect cancer proliferation, ${ }^{7}$ apoptosis, ${ }^{7}$ and differentiation, also to inhibit angiogenesis, ${ }^{8}$ tumor cell invasion, ${ }^{9}$ and metastasis. ${ }^{10}$ Such data propose that EPA and DHA can both make anti-tumor activity potentially representing an effectual adjuvant in cancer chemotherapy and improve some of the secondary difficulties related to cancer, such as cachexia. ${ }^{10,11}$ Evidence exists on the effectiveness of omega-3 polyunsaturated fatty acid DHA as anti-cancer adjuvant, with specific attention to its ability both to increase the uptake of anticancer drugs, particularly in cells otherwise resistant to these drugs, and to enhance the pro-oxidant and pro-apoptotic effectiveness of some chemotherapeutic factors. ${ }^{11}$ This paper investigates the potential consumption of omega-3 polyunsaturated fatty acids EPA and DHA as adjuvant to ameliorate the effectiveness of anticancer treatment. ${ }^{12}$

\section{DHA as adjuvant to ameliorate tumor cell cytotoxicity resulted from oxidative stress}

Some studies demonstrate that high levels of mitochondrial reactive oxygen species (ROS) creation are indispensable for cellular proliferation and tumorigenesis. ROS can influence on target gene expression via phosphorylation, activation and oxidation of transcription factors such as APEX1, NF-kB, P53, and HIF-1 $\alpha .{ }^{13-15}$ Furthermore, ROS can oxidize and suppress signaling molecules like P38 mitogen-activated protein kinase (MAPK) phosphatase, leading to increased proliferation and survival of cancer cells. ${ }^{16}$ Although different anti-cancer drugs initially lead to ROS creation, in most cancer cells the long-term treatment with these drugs decreases the levels of ROS, leading to drug-resistance. ${ }^{17,18}$ Therefore, drug-combination therapies might be a good plan to enhance the effectiveness of common anti-cancer treatments by acting as follows: 1) keeping higher ROS levels in cancer cells, thereby preventing drug resistance; 2) decreasing cancer endogenous antioxidant defense; 3 ) promoting drug uptake and hence apoptosis. ${ }^{11}$

One study indicated DHA dose-dependently promoted reactive species generation and thus membrane lipid peroxidation, in HT29 and Caco-2 colon carcinoma cells. ${ }^{19}$ In addition, some studies indicate that this impact was increased when DHA was administered in combined with some chemotherapeutic factors. It has been demonstrated that in vitro administration of DHA ameliorated adryamicin uptake, cytotoxicity towards L1210 murine leukemia cells and enhanced tumor cell lipid peroxidation and oxidative injury. ${ }^{20}$ Some observations propose that DHA may be able to enhancing the absorption of anticancer drugs in both resistant and sensible cells and that the incorporated DHA-mediated lipid peroxidation may result to greater cytotoxic impact in compared with chemotherapy alone. But, the enhanced drug absorption by DHA was not observed in all tumor cell models. Indeed, though it has been indicated that DHA is able to enhance doxorubicin uptake in doxorubicin resistant mouse leukemia cells $^{21}$ and in MDA-MB-231 and MCF-7dox human breast carcinoma cell lines, the same effect has not been demonstrated in parental MCF7 human breast carcinoma cells. ${ }^{22}$ The inconclusive results might be resulted from the fact that various cell lines might have various cellular features, like membrane formation and configuration. However, even if DHA does not perform in some cells as a drug uptake enhancer, it can make cells more susceptible to oxidative injury made by exogenous factors. Hence, in some cases, DHA can lead to exceed the maximum threshold of ROS tolerability in cancer cells. ${ }^{23}$

Some evidence proposes the proapoptotic effect of DHA, both alone and/or in combined with anti-cancer chemotherapies. ${ }^{11}$ The 
apoptotic impact of DHA seems to happen via both the intrinsic and extrinsic pathways. ${ }^{24}$ This effect is more emphasized by the fact that DHA appears to be a strong inducer of apoptosis only in cancer cells but not in normal cells. For example, it performs as a pro-apoptotic agent in colon cancer cells, while no significant proapoptotic impact was seen in the NCM460 normal human colon mucosal epithelial cell line. ${ }^{25}$ It has been indicated the pro-apoptotic effect of DHA is performed by various ways, consisting of the induction of lipid peroxidation and oxidative stress. Also, DHA can be quickly absorbed by mitochondrial membranes, changing their permeability and reducing the mitochondrial membrane potential. ${ }^{26,27}$ The impacts have been demonstrated in DHA-combined treatment with 5-fluorouracil, oxaliplatin and irinotecan in HT-29 human colorectal adenocarcinoma cells. The anti-cancer effect of DHA, observed in presence of low doses of chemotherapeutic agents, was performed first by loss of mitochondrial membrane potential and then via caspase- 9 activation. ${ }^{28-32}$

\section{Omega-3 polyunsaturated fatty acids as adjuvant to ameliorate the anti-metastatic effectiveness of anticancer therapies}

Tumor metastasis is the main reason of mortality in most cancer patients and hence the most life-threatening aspect facet of various kinds of tumors. Some evidence suggests that the intake of omega-3 polyunsaturated fatty acids and especially DHA impede not only the initiation of many types of tumors but also their development, in that it prevents metastases of murine and human tumors in vivo. ${ }^{33-36}$ In fact, marine fatty acid (EPA and DHA) intake is related to decrease all case mortality in patients with breast cancer. ${ }^{37}$ Moreover, there is some evidence that DHA promotes the anti-metastatic impact of anticancer drugs or other chemical agents. ${ }^{10,38,39}$ In 1994, Rose et al. began certain investigations about the impacts of dietary fish oil omega-3 polyunsaturated fatty acids, on the growth and metastases of MDA-MB-435 human breast cancer cells in female nude mice. ${ }^{40}$ Animals received three isocaloric diets containing 23\% total fat but various ratio of corn oil (rich in linoleic acid) and menhaden oil (rich in DHA and EPA). ${ }^{34,40}$ The researchers found that, in opposite to mice fed diets rich in linoleic acid, mice receiving diets supplemented with DHA or EPA indicated an important inhibition of both primary tumor growth rate and lung metastasis incident and severity proposing a role for DHA and EPA in the suppression not only for tumor growth but also of metastasing of human breast cancer cells. ${ }^{40,41}$ These researchers evaluated tumor responses to DHA or EPA supplementation after surgical excision of the primary tumors. They reported DHA, but not EPA, significantly decreased lung involvement after the surgical excision. ${ }^{42}$ Thus, according to these results, the researchers proposed that omega-3 polyunsaturated fatty acids and especially DHA may have an importance in nutritional therapy of breast cancer as part of both adjuvant and post-excision adjuvant anti-metastatic diet. In another study, by using purified DHA in a murine mammary metastatic tumor model, ${ }^{43}$ the researchers reported that DHA inhibited not only the growth but also metastasis of the MM48 murine mammary tumor transplanted in to $\mathrm{C} 3 \mathrm{H} / \mathrm{He}$ mice. In another investigation, using a mouse model of human breast cancer cell metastasis to bone, it was indicated that dietary fish oil EPA and DHA inhibited the genesis of osteolytic lesions in bone, proposing a useful impact of omega-3 fatty acids EPA and DHA on breast cancer cell metastasis to bone. ${ }^{44}$

The anti-metastatic feature of DHA was also evaluated by use of different animal models of colon cancer metastases. It has been indicated that dietary marine oil (EPA and DHA) prevented the growth and the pulmonary colonization of a transplantable colon tumor implanted in the colon of male Balblc mice. ${ }^{33}$ In another investigation, by use the same subcutaneous implanted highly metastatic colon carcinoma 26 model, it was reported that a DHA rich diet, when administered with tumor cells, notably inhibited lung metastasis. Furthermore, they showed that in vivo DHA-treated tumor cells kept their low potential for lung colony formation when moved to new hosts, suggesting that the impact of DHA was exerted directly on the metastatic capability of tumor cell. ${ }^{45,46}$ Gleissman et al. ${ }^{36}$ demonstrated that DHA, given daily by gavage in atymic rats, retarded the development of established aggressive human neuroblastoma xenografts. The molecular mechanisms by which DHA may influence the metastatic capacity of tumors remain unknown. However, a number of molecular mechanisms have been suggested. Incorporation of DHA in the tumor cell membrane leads to alter in its lipid constitution, which might create plasma membrane notably less fluid and less deformable. ${ }^{47}$ Hence, it has been suggested that the antimetastatic effect of DHA may be associated with noticeable alterations in the fatty acid composition of tumor cell, which damage tumor cell membrane and reduce the capability to metastasize. ${ }^{40,45}$ Mandel et al. by use of the mouse model of human breast cancer cell metastasis to bone indicated that fish oil supplementation significantly suppressed mRNA and protein levels of the cell-surface CD44 adhesion molecule in the MDA-MB-231 tumors, hence recognizing a novel performance for DHA in tumor cells that is the targeting of the cell-intrinsic pro-metastatic CD44 molecule expression. ${ }^{44}$ Some other targets of DHA consisting of cyclooxygenase-2 (COX2), NF-kB, MAPK, peroxisome proliferator activated receptor- $\gamma$ (PPAR- $\gamma$ ), Akt, and B-cell lymphoma/Bcl-2 related X protein (BCL-2/BAX) play a major role in the inhibition of metastases and some reviews are available on their implication in DHA impact on tumor cell proliferation, ${ }^{48}$ angiogenesis,${ }^{8}$ and immune system response, ${ }^{49}$ all crucial events in the metastatic course.

\section{Omega-3 polyunsaturated fatty acids and tumor cell invasion}

In a study about the impact of DHA on tumor cell invasion, Connolly and Rose, by using an in vitro invasion test, evaluated the impact of linoleic acid, EPA, and DHA on the invasion ability of the MDA-MB-435 human breast cancer cell line. They indicated that though all these fatty acids did not impact on the migration of tumor cells, DHA and EPA, but not linoleic acid, significantly suppressed the invasion of tumor cells. ${ }^{29}$ Other researchers evaluated the antiinvasion impact of combining DHA with other nutritional agent such as genistein..$^{10}$ Genistein is an isoflavonoid isolated from soybean, has been demonstrated to exert anticancer effects. These researchers assayed the combination of DHA and genistein for the synergistic suppression of cell invasiveness, inhibition of PGE2 production and COX2 expression in cancer cells. In fact, their data indicated a synergistic impact of genistein and DHA in inhibiting cell invasiveness and endogenous production of PGE2. ${ }^{10}$ The anti-invasive effect of DHA was also evaluated by using other kinds of experimental tumor models. One study indicated that DHA significantly suppressed the invasion of caki-1 cell carcinoma cell line..$^{30}$ In the $70 \mathrm{~W}$ human melanoma cell line (brain metastatic melanoma in nude mice), the researchers indicated DHA reduced invasion and this impact was associated with the suppression of COX2 expression, that consequently downregulated PGE2 production. ${ }^{31}$ Another study reported that DHA prevented migration and invasion of the Bel-7402 human hepatocellular carcinoma cell line. ${ }^{32}$ 


\section{Conclusion}

In conclusion, it seems that combination therapy of omega-3 polyunsaturated fatty acids and antitumor drugs may enhance the cytotoxic effectiveness of drug treatment alone, because it should allow cancer cells to keep higher levels of ROS, decrease endogenous antioxidant tumor cell defenses, and enhance drug uptake. Since the population of cancer patients has had an increasing trend during the last century, finding a useful nutritional intervention to promote the response to treatment with fewer side effects can improve the quality of life in these patients.

\section{Acknowledgments}

This study did not receive any financial support.

\section{Conflicts of interest}

The author declares there is no conflict of interest.

\section{References}

1. Lappe J, Watson P, Travers-Gustafson D, et al. Effect of vitamin D and calcium supplementation on cancer incidence in older women: A randomized clinical trial. Jama. 2017; 317(12):1234-1243.

2. Lake RA, Robinson BW. Immunotherapy and chemotherapy - a practical partnership. Nat Rev Cancer. 2005;5(5):397-405.

3. Solyanik GI. Multifactorial nature of tumor drug resistance. Exp Oncol. 2010;32(3):181-185.

4. Kirkwood JM, Tarhini A, Sparano JA, et al. Comparative clinical benefits of systemic adjuvant therapy for paradigm solid tumors. Cancer Treatment Reviews. 2013;39(1):27-43.

5. Pritchard JR, Lauffenburger DA, Hemann MT. Understanding resistance to combination chemotherapy. Drug Resist Updat. 2012;15(5-6):249257.

6. Vaughan VC, Hassing MR, Lewandowski PA. Marine polyunsaturated fatty acids and cancer therapy. British Journal of Cancer. 2013;108(3):486492.

7. Chamras H, Ardashian A, Heber D, et al. Fatty acid modulation of MCF7 human breast cancer cell proliferation, apoptosis and differentiation. $J$ Nutr Biochem. 2002;13(12):711-716.

8. Spencer L, Mann C, Metcalfe M, et al. The effect of omega-3 FAs on tumour angiogenesis and their therapeutic potential. Eur $J$ Cancer. 2009;45(12):2077-2086.

9. D’Eliseo, Manzi L, Merendino N, et al. Docosahexaenoic acid inhibits invasion of human RT112 urinary bladder and PT45 pancreatic carcinoma cells via down-modulation of granzyme B expression. Journal of Nutritional Biochemistry. 2012;23(5):452-457.

10. Horia E, Watkins BA. Complementary actions of docosahexaenoic acid and genistein on COX-2, PGE2 and invasiveness inMDA-MB-231 breast cancer cells. Carcinogenesis. 2007;28(4):809-815.

11. Siddiqui RA, Harvey KA, Xu Z, et al. Docosahexaenoic acid: a natural powerful adjuvant that improves efficacy for anticancer treatmentwith no adverse effects. Biofactors. 2011;37(6):399-412.

12. Colomer R, Moreno-Nogueira JM, Garcia-Luna PP, et al. N-3 fatty acids, cancer and cachexia: a systematic review of the literature. British Journal ofNutrition. 2007;97(5):823-831.

13. Horak P, Crawford AR, Vadysirisack DD, et al. Negative feedback control of HIF-1 through REDD1-regulated ROS suppresses tumorigenesis. Proc Natl Acad Sci USA. 20109;107(10):4675-4680.
14. Chattopadhyay R, Das S, Maiti AK, et al. Regulatory role of human APendonuclease (APE1/Ref-1) in YB-1-mediated activation of the multidrug resistance gene MDR1. Mol Cell Biol. 2008;28(23):7066-7080.

15. Gottlieb E, Vousden KH. P53 regulation of metabolic pathways. Cold Spring Harbor Perspectives in Biology. 2010;2(4):001040.

16. Wang X, Liu JZ, Hu JX, et al. ROS-activated p38 MAPK/ERK-Akt cascade plays a central role in palmitic acid stimulated hepatocyte proliferation. Free Radic Biol Med. 2011;51(2):539-551.

17. Maiti AK. Gene network analysis of oxidative stress-mediated drug sensitivity in resistant ovarian carcinoma cells. Pharmacogenomics $J$. 2010;10(2):94-104.

18. Murphy RA, Mourtzakis M, Chu QS, et al. Supplementation with fish oil increases first-line chemotherapy efficacy in patients with advanced nonsmall cell lung. Cancer. 2011;117(16):3774-3780.

19. Hossain Z, Hosokawa M, Takahashi K. Growth inhibition and induction of apoptosis of colon cancer cell lines by applying marine phospholipid. Nutrition and Cancer. 2008;61(1):123-130.

20. Guffy MM, North JA, Burns CP. Effect of cellular fatty acid alteration on adriamycin sensitivity in cultured L1210 murine leukemia cells. Cancer Research. 44(5):1863-1866.

21. Liu QY, Tan BKH. Effects of cis-unsaturated fatty acids on doxorubicin sensitivity in P388/DOX resistant and P388 parental cell lines. Life Sciences. 2000;67(10):1207-1218.

22. Mahéo K, Vibet S, Steghens JP, et al. Differential sensitization of cancer cells to doxorubicin by DHA: a role for lipoperoxidation. Free Radic Biol Med. 2005;39(6):742-751.

23. Pelicano H, Carney D, Huang P. Mechanisms and biological relevance of lipid peroxidation initiation. Chemical Research in Toxicology. 1993;6(1):2-18.

24. Serini S, Piccioni E, Merendino N, et al. Dietary polyunsaturated fatty acids as inducers of apoptosis: implications for cancer. Apoptosis. 2009;14(2):135-152.

25. Engelbrecht AM, Toit-Kohn JL, Ellis B, et al. Differential induction of apoptosis and inhibition of the PI3-kinase pathway by saturated, monounsaturated and polyunsaturated fatty acids in a colon cancer cell model. Apoptosis. 2008;13(11):1368-1377.

26. Suzuki H, Manabe S, Wada O, et al. Rapid incorporation of docosahexaenoic acid from dietary sources into brain microsomal, synaptosomal andmitochondrial membranes in adult mice. Int $J$ Vitam Nutr Res. 1997;67(4):272-278.

27. Di Paola M, Lorusso M. Interaction of free fatty acids with mitochondria: coupling, uncoupling and permeability transition. Biochim Biophys Acta. 2006;1757(9-10):1330-1337.

28. Granci V, Cai F, Lecumberri E, et al. Colon cancer cell chemosensitisation by fish oil emulsion involves apoptotic mitochondria pathway. British Journal of Nutrition. 2013;109(7):1188-1195.

29. Connolly JM, Rose DP. Effects of fatty acids on invasion through reconstituted basement membrane ("Matrigel") by a human breast cancer cell line. Cancer Letters. 1993;75(2):137-142.

30. McCabe AJ, Wallace JMW, Gilmore WS, et al. Docosahexaenoic acid reduces in vitro invasion of renal cell carcinoma by elevated levels of tissue inhibitor of metalloproteinase-1. Journal of Nutritional Biochemistry. 2005;16(1):17-22.

31. Denkins Y, Kempf D, Ferniz M, et al. Role of $\omega-3$ polyunsaturated fatty acids on cyclooxcyclooxygenase-2 metabolism in brain-metastatic melanoma. J Lipid Res. 2005;46(6):1278-1284. 
32. Sun SN, Jia WD, Chen H, et al. Docosahexaenoic acid (DHA) induces apoptosis in human hepatocellular carcinoma cells. Int J Clin Exp Pathol. 2013;6(2):281-289.

33. Broitman SA, Cannizzo F. Amodel systemfor studying nutritional interventions on colon tumor growth: effects of marine oil. Advances in ExperimentalMedicine and Biology. 1992;322:103-118.

34. Rose DP, Connolly JM, Rayburn J, et al. Influence of diets containing eicosapentaenoic or docosahexaenoic acid on growth and metastasis of breast cancer cells in nude mice. J Natl Cancer Inst. 1995;87(8):587-592.

35. Yam D, Peled A, Huszar M, et al. Dietary fish oil suppresses tumor growth and metastasis of Lewis lung carcinoma in mice. Journal of Nutritional Biochemistry. 1997;8(11):619-622.

36. Gleissman H, Segerström L, Hamberg M, et al. Omega-3 fatty acid supplementation delays the progression of neuroblastoma in vivo. Int $J$ Cancer. 2011;128(7):1703-1711.

37. Patterson RE, Flatt SW, Newman VA. Marine fatty acid intake is associated with breast cancer prognosis. Journal of Nutrition . 2011;141(2):201-206.

38. Yam D, Peled A, Shinitzky M. Suppression of tumor growth and metastasis by dietary fish oil combined with vitamins $\mathrm{E}$ and $\mathrm{C}$ and cisplatin. Cancer Chemotherapy and Pharmacology. 2001;47(1)34-40.

39. Bougnoux P, Hajjaji N, Ferrasson MN, et al. Improving outcome of chemotherapy of metastatic breast cancer by docosahexaenoic acid: a phase II trial. Br J Cancer. 2009;101(12):1978-1985.

40. Rose DP, Connolly JM . Effects of dietary fish oil on fatty acids and eicosanoids in metastasizing human breast cancer cells. J Natl Cancer Inst. 1993;85(21):1743-1747.

41. Connolly JM, Liu XH, Rose DP. Effects of dietarymenhaden oil, soy, and a cyclooxygenase inhibitor on human breast cancer cell growth and metastasis in nude mice. Nutr Cancer. 1997;29(1):48-54.
42. Rose DP, Connolly JM, Coleman M. Effect of omega-3 fatty acids on the progression of metastases after the surgical excision of human breast cancer cell solid tumors growing in nude mice. Clin Cancer Res. 1996;2(10):1751-1756.

43. Kinoshita K, Noguchi M, Tanaka M. Effects of linoleic acid, eicosapentaenoic acid, and docosahexaenoic acid on the growth and metastasis of MM48 mammary tumor transplants in mice. International Journal of Oncology. 1996;8(3):575-581.

44. Mandal CC, Ghosh-Choudhury T, Yoneda T, et al. Fish oil prevents breast cancer cell metastasis to bone. Biochem Biophys Res Commun. 2010;402(4):602-627.

45. Iigo $M$, Nakagawa $T$, Ishikawa $C$, et al. Inhibitory effects of docosahexaenoic acid on colon carcinoma 26 metastasis to the lung. British Journal of Cancer. 1997;75(5)650-655.

46. Suzuki I, Iigo M, Ishikawa $C$, et al. Inhibitory effects of oleic and docosahexaenoic acids on lung metastasis by colon carcinoma-26 cells are associated with reduced matrix metalloproteinase- 2 and -9 activities. International Journal of Cancer. 1997;73(4)607-612.

47. Zerouga M, Jenski LJ, Booster S, et al. Can docosahexaenoic acid inhibit metastasis by decreasing deformability of the tumor cell plasma membrane? Cancer Letters. 1997;119(2)163-168.

48. Cockbain AJ, Toogood GJ, Hull MA. Omega-3 polyunsaturated fatty acids for the treatment and prevention of colorectal cancer. Gut. 2012;61(1):135-149.

49. Calder PC. Long-chain fatty acids and inflammation. Proceedings of the Nutrition Society. 2012;71(2):284-289. 